\title{
Exploring the Effect of Word-Scale Visualizations on Reading Behavior
}

\author{
Pascal Goffin \\ Inria, Aviz \\ Bat 660, Univ Paris-Sud \\ F91405 ORSAY Cedex, France F91405 ORSAY Cedex, France \\ pascal.goffin@inria.fr \\ Anastasia Bezerianos \\ U.Paris-Sud, CNRS\&Inria, Ilda \\ Bat 660, Univ Paris-Sud \\ anastasia.bezerianos@lri.fr
}

\author{
Wesley Willett \\ Inria, Aviz \\ Bat 660, Univ Paris-Sud \\ Petra Isenberg \\ Inria, Aviz \\ Bat 660, Univ Paris-Sud \\ F91405 ORSAY Cedex, France F91405 ORSAY Cedex, France \\ wj@wjwillett.net \\ petra.isenberg@inria.fr
}

Permission to make digital or hard copies of part or all of this work for personal or classroom use is granted without fee provided that copies are not made or distributed for profit or commercial advantage and that copies bear this notice and the full citation on the first page. Copyrights for third-party components of this work must be honored. For all other uses, contact the Owner/Author. Copyright is held by the owner/author(s).

CHI'15 Extended Abstracts, April 18-23, 2015, Seoul, Republic of Korea. ACM 978-1-4503-3146-3/15/04.

http://dx.doi.org/10.1145/2702613.2732778

\begin{abstract}
We studied how the integration of small visualizations (wordscale visualizations) into a sentence affects reading speed and memorization during a brief reading task. In particular we were interested in how different placement types-with their inherent text appearance and layout changes-affect readers. We designed a quantitative study in which we gave sentences with or without visualizations for participants to read. Then, we invited them to answer questions on the sentences. We found that the information encoded in the visualizations is more prominent and easily remembered than information in the written text, but that different placement options had little to no effect on reading performance, even if participants had different preferences.
\end{abstract}

\section{Author Keywords}

Information visualization; Text visualization; Glyphs; WordScale visualizations; Sparklines; Readability

\section{ACM Classification Keywords}

H.5.m [Information interfaces and presentation (e.g., $\mathrm{HCl}$ )] Miscellaneous.

\section{Introduction}

We explore how inserting small visualizations, called wordscale visualizations, into text affects readers. These visualizations display information associated with specific words 
ezirkshauptmannschaft Dux

a) ‡andant Robert Jacobi was on

duties by a control of the

zirkshauptmannschaft Dux

b) 喜 idant Robert Jacobi In_-

$y$ in his duties by a control of

'ezirkshauptmannschaft Dux

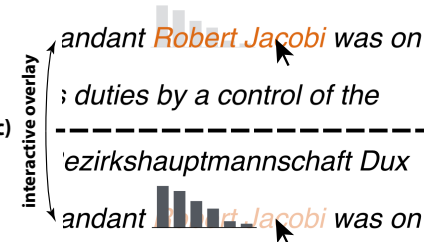

; duties by a control of the

'ezirkshauptmannschaft Dux

d) $\sum_{0}^{\bar{n}}$ andant Robert Jacobi was on

; duties by a control of the

Figure 1: The four word-scale visualization positions: (a) "top", (b) "right", (c) interactive "overlay" (d) "no word-scale visualization" (no wsv). in the text, and are a generalization of the more well-known term sparkline: "a small, intense, simple, word-sized graphic with typographic resolution" [11]. Word-scale visualizations can use a variety of visual encodings and can encompass a wider range of "word-scales", including sizes larger than a word, but usually smaller than a sentence or paragraph [7]

One of the most common application contexts for word-scale visualizations is text documents. Embedded in document text, word-scale visualizations can deliver additional detailed information associated with specific words or phrases e.g visualizations of recent stock performance next to the name of a company, or small maps next to the name of a country. Integrating these small visualizations in a text [11] can be useful in cases where the raw information is not crucial for a first read, or has too many dimensions and values to express in words without boring the reader or bloating the text.

In our past work [7], we charted a design space of placement options for word-scale visualizations in text documents. We demonstrated how different placement options like placing visualizations between words or between lines of text can drastically affect the text layout. Inserting word-scale visualizations between words or lines may require to reflow the text, to add inter-line space or to increase the space between words. Given that the placement of word-scale visualizations can significantly change the layout and appearance of a text, we hypothesize that readers may react differently to the text depending on what placement strategy is used. It is possible that introducing word-scale visualizations above the entity in the interline space (Figure 1a), to the right of the entity (Figure $1 \mathrm{~b}$ ), or when using interaction to reveal a hidden word-scale visualization (Figure $1 \mathrm{c}$ ) may impact reading behavior in different ways because they disrupt the text in very different ways. While prior work has described use cases for word-scale visualizations $[7,11]$, it is not clear how their integration affects reading the text. In order to address this question, we conducted an initial study that examines how placing word-scale visualizations affects reading speed retention, and whether readers tend to use information from the word-scale visualizations or the text.

Our results show no impact of placement options on reading performance. However, information encoded in the wordscale visualizations was often more prominent and easily remembered than information in the written text.

\section{Related Work}

We recently defined word-scale visualizations [7] as more general sparklines [11] (see Introduction). In our past work [7] we also showed several examples of word-scale visualizations and how they can be embedded into different positions in text documents. In addition to these examples, Gestaltlines [1] and SportLines [9] are recently published word-scale visualizations from other authors.

Yet, while examples exist, no prior studies have explored the effects on readers when word-scale visualizations are embedded in text. Related work, however, has considered other additions to text documents and their effect on readbility. For example, Diaper and Waelend [5] suggest that graphics surrounding blocks of text do not distract readers of web pages. Zellweger et al. [12] conducted an observational study using an eye tracker to study the addition of textual annotations to their Fluid Documents [3]. They used several different placement conditions including the inter-line space, margins, and pop-ups. They did not find any significant differences in eye movement between conditions but subjective preferences were complex and depended on many different factors. Some participants preferred having the complete static text for the additional information always visible. Other participants preferred the possibility 
Ants in Islandia-State had a suicide mortality rate

per one hundred thousand ants of 6.0 , with the

suicide rate increasing over the years.

$\square$ Trend

Figure 2: An example sentence describing information is ambiguously encoded in the sentence (trend = increasing) and in the word-scale visualization (decreasing). Colors added for illustration.

The average class size of a primary school class in Drusselstein was 90 ant students, although th class size has been decreasing over the years.

\section{$\square$ Statisicic (Indicator)}

$\square$ Colony

Figure 3: An example sentence for an ant colony with the statistic "average class size" characterized by a number and a trend over the last 50 ant years. Colors added for illustration. to change the spatial arrangements of the primary text on demand to reveal the extra text. This study relates to ours as we similarly include a static and dynamic condition for viewing word-scale visualizations. Yet, both previous studies differ from ours as they did not study graphic visualizations that require a different type of reading compared to text or graphical icons.

\section{Experiment}

Our long-term goal is to study how the placement of wordscale visualizations in text impacts reading in a broader context. We intend to conduct a series of studies and here report on our first one that examined how word-scale visualizations are used by readers in a timed information seeking task. We assessed if the position of word-scale visualizations has an impact on reading and retention of information from a short sentence. Additionally, we checked whether participants preferred to retrieve information from the text or from the word-scale visualizations.

\section{Factors}

The study factors were the four main word-scale visualization positions: "top", "right", interactive "overlay" and "no word-scale visualization" (Figure $1(\mathrm{a}, \mathrm{b}, \mathrm{c}, \mathrm{d})$ ) from our previously published design space [7]

Each sentence given to the participants belonged to one of five groups: The first group consisted of sentences without word-scale visualizations. The four remaining groups had one word-scale visualization per sentence. Each sentence contained information that had to be used to answer questions after reading. For the cases that contained visualizations we explored four different information-related conditions-a) the information was encoded only in the text, b) the information was encoded only in the visualization, c) the information was redundantly encoded in both the text and the visualization, d) information was encoded in text and visualization but contradicted each other. The goal of the last case d) (Figure 2) was to see from where readers took the information to answer a post-reading question.

Task

Each Participant's task was to read and remember information from a single sentence. We concentrated on single sentences as opposed to smaller texts or paragraphs to build up our knowledge on the effects of word-scale visualizations on reading from small textual building blocks. Sentences are also a good unit to measure the speed of reading. Future studies-informed by this one-will include longer texts.

\section{Procedure}

We gave participants initial written instructions explaining word-scale visualizations and the study procedure. We then presented reading tasks to participants in blocks, where each participant saw all tasks for a given presentation position (top, right, overlay or no word-scale visualization) before moving on to the next placement. This was done using a $4 \times 4$ Latin square design. At the beginning of each block we included a small training ( 3 sentences) to familiarize the participants with the new position.

The dataset used was about a fictional ant empire with different colonies. Each sentence provided a statistic (e.g. education expenses, life expectancy) about one of the colonies at a specific point in time with an associated quantity and a trend description (Figure 3 ).

First the sentence (with or without word-scale visualization) was shown. When the participant was done reading, they had to press SPACE to advance to questions on the sentence. Answering them and pressing SPACE concluded a trial. At the end of the study, participants filled out a questionnaire about their reading strategy and preferences concerning 
What was the general trend mentioned? $\mathrm{O}$ upward trend

O downward trend

O constant trend

$\mathrm{O}$ up-and-down trend

Oambiguous

Which rate is the sentence about?

$O$ birth mortality rate

$\mathrm{O}$ accident mortality rate

$\bigcirc$ life expectancy rate

suicide mortality rate

Figure 4: The two multiple choice questions with four potential answers for the sentence in Figure 2. The first question asks for the trend. The second question ask for the statistic.

\section{word-scale visualization positioning.}

We instructed participants to read the sentence and to answer the questions as quickly as possible. The questions consisted of two multiple choice questions each with four potential answers (Figure 4). The first multiple choice question asked either about the trend seen in the word-scale visualization for the cases where one was present, or some other information (colony, number or statistic). The second question asked either about the colony, the statistic, or the number present in the sentence. The goal of this second control question was to see how well the sentence was read and how well the relevant information was retrieved from the sentence.

\section{Measures}

We used two measures of performance: sentence reading time and error rate. Sentence reading time was the interval between sentence presentation and the moment the participant pressed the SPACE bar to advance to the questions.

We measured the error rate for the trend question for the sentences without word-scale visualizations and the sentences with word-scale visualizations where the information was either present only in the sentence, or only in the wordscale visualization, or placed redundantly in both sentence and word-scale visualization. For these cases the answer was either right or wrong. For the case where information was ambiguously encoded we differentiated between answers that were wrong, came from the sentence, came from the word-scale visualization, or if the participant correctly reported the inconsistency.

\section{Participants}

We recruited twelve (four females) participants with their age ranging from $20-30$. They were all $\mathrm{PhD}$ students at the Computer Science department and had expertise in Human-
Computer Interaction $(\mathrm{HCl})$ and Information Visualization (InfoVis). One had minor dyslexia.

Hypotheses

Our analysis was driven by three main hypotheses linked to reading time and error rate.

(H1) Reading a sentence with a word-scale visualization in creases reading time. Comparing individual positions, "top" takes more time than "right" since readers can no longer continue their linear reading flow. "Overlay" needs longest as interaction is involved

(H2) Questions for sentences with a word-scale visualization will be answered more correctly given the memorable visual nature of the word-scale visualization.

(H3) Readers will most often use information from the word-scale visualization to answer questions for ambiguous cases given their memorable visual nature.

Data Analysis

With respect to the concerns and recommendations regarding null-hypotheses testing $[4,6]$ we base our analysis and discussions on estimation, i.e., we use effect sizes with confidence intervals. All point estimates and $95 \% \mathrm{Cl}$ are based on 1000 percentile bootstrap replicates of the statistic applied to the data [2].

We log-transformed the time measures to correct for skewness [10]. All reported means are anti-logged and therefore indicate geometric means [10].

\section{Results}

We first report on performance results and then move on to data from the questionnaire. 


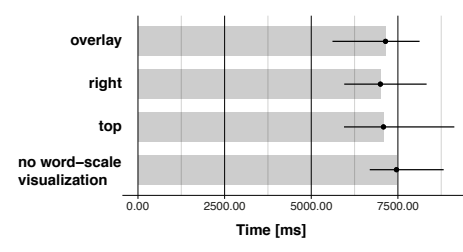

Figure 5: $95 \% \mathrm{Cl}$ for participants time across placement positions.

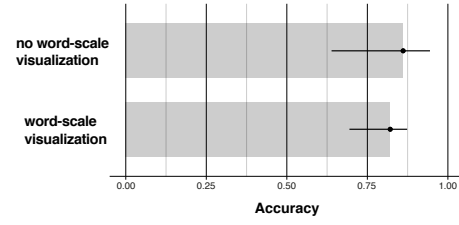

Figure 6: $95 \% \mathrm{Cl}$ for the accuracy for sentences with and without word-scale visualization (in the non-ambiguous cases).

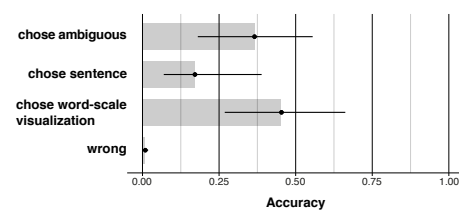

Figure 7: $95 \% \mathrm{Cl}$ for people's choices in the ambiguous case.

\section{Performance Results}

Surprisingly, we were unable to confirm $\mathrm{H} 1$ that placement had an effect on reading performance. Figure 5 is a breakdown of the sentence reading time per placement. The fastest placement position was "right" with a GM of 7s $(\mathrm{Cl}[6,8.3])$. Yet, the difference to the slowest placement position "no word-scale visualization" (GM 7.46s; Cl [6.7, 8.5]) was only $0.46 \mathrm{~s}$ - too small to likely make a practical difference. It is particularly interesting to see that interaction had no practical detrimental effect with the "overlay" condition's $\mathrm{GM}$ at $7.14 \mathrm{~s}(\mathrm{Cl}[5.6,8.1])$.

We could also not confirm H2. Figure 6 shows a breakdown of the accuracy rates for the two cases: "word-scale visualization" and "no word-scale visualization". No clear difference between the two cases is evident. The difference between the case "word-scale visualization" (GM 0.82s; $\mathrm{Cl}$ $[0.69,0.87])$ and the case "no word-scale visualization" (GM $0.86 \mathrm{~s} ; \mathrm{Cl}[0.64,0.94])$ was only $0.04 \mathrm{~s}$-also too small to likely make a practical difference.

Figure 7 is a breakdown of the accuracy rate for participant's choices with respect to the ambiguous case. Often participants recognized the ambiguity (GM 0.37; $\mathrm{Cl}[0.18,0.56])$, thus they had looked at and remembered information from both the sentence and the word-scale visualization. When participants did not identify the ambiguity, they tended to pull their answer most often from the word-scale visualization (GM 0.45; $\mathrm{Cl}[0.27,0.66])$ rather than the sentence (GM $0.17 ; \mathrm{Cl}[0.07,0.39]$ ). This partially confirms $\mathrm{H} 3$, in that when readers did not spot the ambiguities word-scale visualizations were used more.

\section{Questionnaire Results}

We summarized the ranking information from the questionnaire in Figure 8. We removed one participant from the pool of questionnaire responses as this participant did not understand the question concerning ranking.

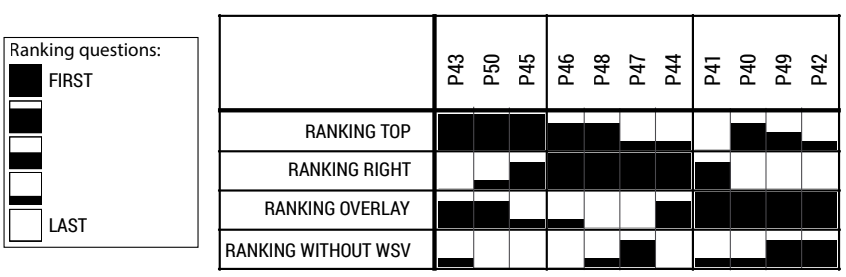

Figure 8: Summarized questionnaire data from Bertifier [8]: Grouped participant responses w.r.t. ranking for each position.

We asked participants to rank the four word-scale visualizations positions according to their personal preference. In Figure 8 "the more black" in the cell, the higher a position was ranked. We can distinguish three groups of participants: Three participants ranked the "top" position first, four ranked the "right" position first, and another four ranked the interactive "overlay" position first. Given combined ranking results, the "top" position was most preferred, while the "right" position was the least popular together with the "no word-scale visualization" position.

No position was consistently ranked number 1 , for different reasons explained by participants. P43 said in favor of "top": "I prefer the "top" word-scale visualization over the "interactive" one because it provides the best of two worlds: allows for reading smoothly and allows for quick access to trend with one glance. It works for this particular example (short text)." P44 said in favor of "right": "... right of the word was the easiest for me as I just had to follow the line to get the information ...". P42 preferred "overlay": "[The] interactive version gave me the choice to display [only when] I felt the need ...". There were also negative comments regarding different positions. E. g. P49 said: "I disliked the right position both because it forced me to stop my reading and also for aesthetics reasons". 


\section{Discussion and Conclusions}

When combining the data from the measurements of sentence reading time and error rate and the data from our questionnaire no single position emerges as the best. Additionally, no single position was consistently preferred by all participants. This may actually be a positive result, as it suggests that a variety of word-scale visualization positions can be experimented with in the future for different use cases. It may even be appropriate to allow readers to choose where they want the word-scale visualizations to be placed, seeing as our participants voiced strong preferences for several different positions.

As revealed through our first study results we believe that integrating word-scale visualizations into text to provide context or additional information for specific words remains an interesting subject to pursue. Participants were drawn to the word-scale visualization and seemed to use the information in the word-scale visualization more than the information in the sentence in cases where inconsistencies in the two were not spotted.

We are curious to confirm our results and also see how readers will deal with word-scale visualizations in more substantial texts. Additionally, we are interested in whether or not word-scale visualizations can enhance memorability and text comprehension. Another direction to pursue is to investigate which cases and contexts are more suitable to which position.

\section{Acknowledgments}

We would like to thank our study participants for their valued feedback and their participation. This work is sponsored by the French Research Organization, project grant ANR-11JS02-003 and supported by the Collaborative European Digital Archive Infrastructure project CENDARI (cendari.eu).

\section{References}

[1] Brandes, U., Nick, B., Rockstroh, B., and Steffen, A. Gestaltlines. Computer Graphics Forum 32, 3 (2013), 171-180.

[2] Canty, A., and Ripley, B. Bootstrap Functions. 2014.

[3] Chang, B.-W., Mackinlay, J. D., Zellweger, P. T., and Igarashi, T. A negotiation architecture for fluid documents. In Proc. UIST, ACM (1998), 123-132.

[4] Cumming, G. The new statistics: Why and how. Psychological science 25, 1 (2014), 7-29.

[5] Diaper, D., and Waelend, P. World wide web working whilst ignoring graphics: good news for web page designers. Interacting with computers 13, 2 (2000), 163-181.

[6] Dragicevic, P., Chevalier, F., and Huot, S. Running an hci experiment in multiple parallel universes. In $\mathrm{CHI}$ Extended Abstracts, ACM (2014), 607-618.

[7] Goffin, P., Willett, W., Fekete, J.-D., and Isenberg, $P$. Exploring the placement and design of word-scale visualizations. IEEE TVCG 20, 12 (2014), 2291-2300.

[8] Perin, C., Dragicevic, P., and Fekete, J.-D. Revisiting bertin matrices: New interactions for crafting tabular visualizations. IEEE TVCG 20, 12 (2014), 2082-2091.

[9] Perin, C., Vuillemot, R., and Fekete, J.-D. SoccerStories: A kick-off for visual soccer analysis. IEEE TVCG 19, 12 (2013), 2506-2515.

[10] Sauro, J., and Lewis, J. R. Average task times in usability tests: what to report? In Pro. CHI, ACM (2010), 2347-2350.

[11] Tufte, E. R. Beautiful Evidence. Graphics Press, Cheshire, CT, 2006.

[12] Zellweger, P. T., Regli, S. H., Mackinlay, J. D., and Chang, B.-W. The impact of fluid documents on reading and browsing: An observational study. In Proc. CHI, ACM (2000), 249-256. 